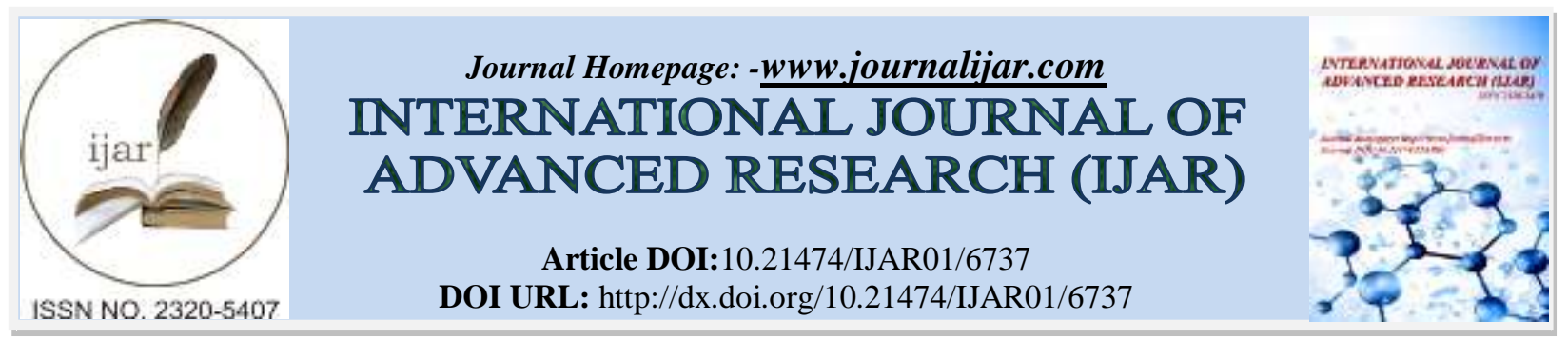

RESEARCH ARTICLE

\title{
COMPARATIVE EVALUATION OF HARDNESS OF DIFFERENT RESTORATIVE MATERIALS (RESTORATIVE GIC, CENTION N, NANOHYBRID COMPOSITE RESIN AND SILVER AMALGAM) - AN IN VITRO STUDY.
}

\section{Paromita Mazumdar ${ }^{1}$, Abiskrita Das ${ }^{2}$ and Chiranjan Guha ${ }^{3}$.}

1. Professor and H.O.D, Department of Conservative Dentistry \& Endodontics, Guru Nanak Institute of Dental Sciences and Research, 157/F Nilgunj Road, Panihati, Kolkata-700114

2. Post Graduate student, Department of Conservative Dentistry \& Endodontics, Guru Nanak, Institute of Dental Sciences and Research, 157/F, Nilgunj Road, Panihati, Kolkata-700114.

3. Post Graduate student, Department of Conservative Dentistry \& Endodontics, Guru Nanak, Institute of Dental Sciences and Research, 157/F, Nilgunj Road, Panihati, Kolkata-700114.

\section{Manuscript Info}

Manuscript History

Received: 13 January 2018

Final Accepted: 15 February 2018

Published: March 2018

Keywords:-

Microhardness, Amalgam; Cention N,

Glass ionomer cement.

\section{Abstract}

Aim: To evaluate the hardness of four restorative materials, nanohybrid composite resin, Cention $\mathrm{N}$, silver amalgam and type II GIC

Materials and method: With the help of a $5 \mathrm{~mm}$ diameter straw, already cut to $3 \mathrm{~mm}$ in length, cylindrical plastic moulds were prepared. Restorative materials were inserted in different moulds Composite resin and cention $\mathrm{N}$ were polymerized with LED. Moulds filled with materials were covered with a glass slab to provide a flat surface. All the samples were stored in distilled water for $24 \mathrm{hrs}$. The samples so prepared were divided into groups as follows and subjected to experimentation. The samples were randomly tested with microardness indentor.

Result: Among all the restorative materials, Cention N showed highest microhardness value followed by silver amalgam, nanohybrid composite resin and type II glass ionomer cement

Conclusion: According to our results, the microhardness of restorative materials could withstand the masticatory forces in the clinical context. Cention $\mathrm{N}$ showed better microhardness properties becoming a more clinically suitable option for minimal invasive treatments.

Copy Right, IJAR, 2018,. All rights reserved.

\section{Introduction:-}

Numerous direct filling materials are available to the modern dental practice - from amalgams through to modern bulk fill composites. Amalgam materials were first introduced to western dentistry in the 19th century, Glass ionomer cements (GICs) were introduced around the 1970s, Composite resins became standard during the 1980s, Resin modified glass ionomers and compomers were introduced in the 1990s and the current decade saw the launch of several bulk-fill composites. [1] 
Amalgams and glass ionomer cements can be viewed as basic filling materials. Basic in the sense that they are longestablished, economical and simple to use. They are usually applied in bulk without an adhesive, are self-curing and do not require complicated dental equipment.

Great strides forward in direct filling materials have been made with dental composites and their accompanying adhesives in recent decades; simple, basic restoratives such as amalgam and glass ionomer cements remain popular.[1] Evolutionary development of filling materials leads to an increasing need for better tooth colored restorative materials to replace missing tooth structure and to modify tooth color and contour, thus enhance facial esthetics. Polymeric restoratives have continued to evolve into the direct restorative materials of choice mainly because of their superior aesthetic characteristics.[2]

Currently, composites are the most widely used materials in restorative dentistry. Composites consist of a mixture of two or more materials. Each of these materials contributes to the overall properties of the composite.[3] Dentists have long sought after a real alternative to amalgam or glass ionomer cements - a cost-effective, fluoride releasing product that is quick and easy to use without complicated equipment and that offers both strength and good esthetics. [1]

Cention $\mathrm{N}$ is a tooth-coloured, basic filling material for direct restorations. It is self-curing with optional additional light-curing. The alkasite Cention $\mathrm{N}$ thus redefines the basic filling, combining bulk placement, ion release, and durability in a dual-curing, esthetic product - satisfying the demands of both dentists and patients. [1]

Physical characteristics are of critical importance when deciding on suitable materials, because they strongly influence the clinical durability of restorations.

One of the most important property is the material hardness, which correlates well to compressive strength, abrasion resistance, and degree of conversion. [4]

Hardness is defined as the resistance of a material to indentation or penetration. It has been used to predict the wear resistance of a material and its ability to abrade or be abraded by opposing tooth structures. [5]

Low hardness values are usually linked to poor wear resistance and susceptibility to scratching, which can compromise fatigue strength and lead to restoration failures. [6]

The aim of the study was to evaluate the hardness of nanohybrid composite resin, Cention N, silver amalgam and type II GIC

\section{Materials and method:-}

Restorative procedures:-

All the prepared samples were divided into 4 experimental groups, with 10 teeth in each group according to the restorative material used: Group I- Nanohybrid composite resin; Group II-Cention N; Group III- Silver Amalgam, Group IV- Type II GIC

\section{Study design:-}

\section{Sample design:-}

With the help of a $5 \mathrm{~mm}$ diameter straw, already cut to $3 \mathrm{~mm}$ in length, cylindrical plastic moulds were prepared. Materials were inserted in different moulds with the help of a plastic filling instrument

The samples so prepared were divided into groups as follows and subjected to experimentation.

The 40 samples so prepared were divided into groups as follows and subjected to experimentation.

\section{Group I:-}

Type II glass ionomer cement (GC lot no. 1612101) was placed in the mould using a plastic filling instrument.

\section{Group II:-}

Cention N (Ivoclar Vivadent lot no. V26429) was placed in the mould using a plastic filling instrument and condensed with the help of a condenser 


\section{Group III:-}

Nanohybrid composite resin (Ivoclar Vivadent was placed in the mould using a plastic filling instrument and condensed with the help of a condenser

\section{Group IV:-}

The silver amalgam (DPI batch no. 2171) was placed in the mould using the amalgam carrier and condensed with the help of amalgam condenser without a liner.

Composite resin and cention $\mathrm{N}$ were polymerized with LED unit. Moulds filled with materials were covered with a glass slab to provide a flat surface. All the samples were stored in distilled water for $24 \mathrm{hrs}$ at $37^{\circ} \mathrm{C}$.

The specimens were stored in distilled water for $24 \mathrm{hrs}$. The samples were randomly tested with microhardness indenter. Microhardness indentor were used to make five indentation in the centre of the surface. Mean of the microhardness were taken by Vicker's hardness tester

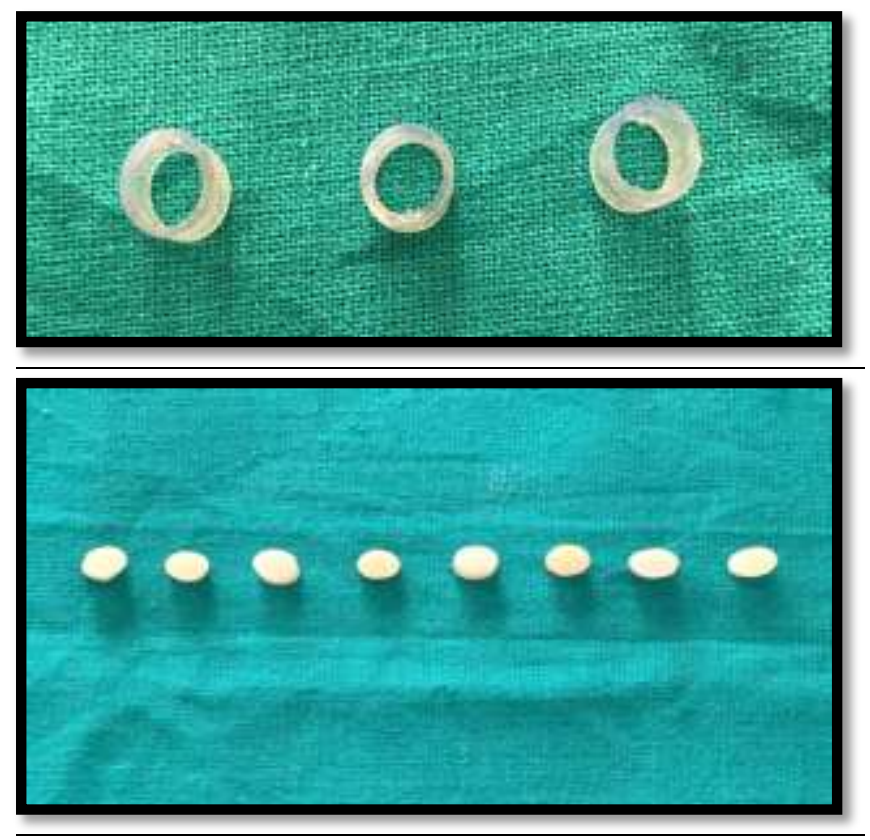

\section{Vicker's Hardness Test:-}

The microhardness indenter started in the center of the sample and three indentations linear to the four cardinal points with a distance of $4 \mathrm{~mm}$ between each other. [7]

For the calculation of Vickers microhardness (VHN), the lengths of the two diagonals of each indentation were measured and VHN was calculated using the following formula:

$\mathrm{VHN}=1.854 \mathrm{~F} / \mathrm{d} 2$

Where $\mathrm{F}$ is the load applied in Newtons and $\mathrm{d}$ is the mean length of the two diagonals of each indentation. 

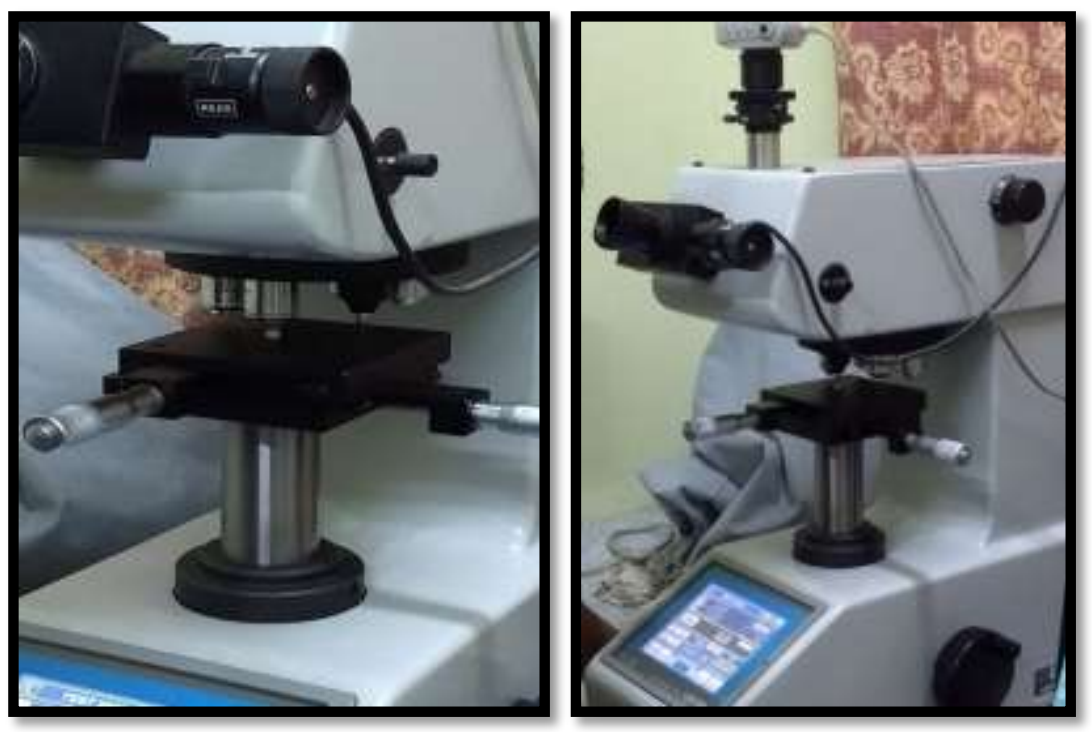

\section{Statistical Analysis:-}

The mean value and standard deviation were calculated. VHN data were subject to ANOVA. All data were analyzed with SPSS. Significant differences were considered at $\mathrm{p}<0.05$. All the statistical analysis was performed

\section{Result:-}

In this experimental and comparative in vitro study, all data showed normal distribution. The mean values of Vickers hardness are summarized in Table 2. The Cention N exhibit the highest VHN (Harder) followed by silver amalgam, nanohybrid composite resin and type II glass ionomer cement.

Cention $\mathrm{N}$ resulted in higher $(\mathrm{p}<0.01)$ hardness than the other restoarative materials. Silver amalgam resulted in higher $(\mathrm{p}<0.05)$ hardness than nanohybrid composite resin and type II GIC.

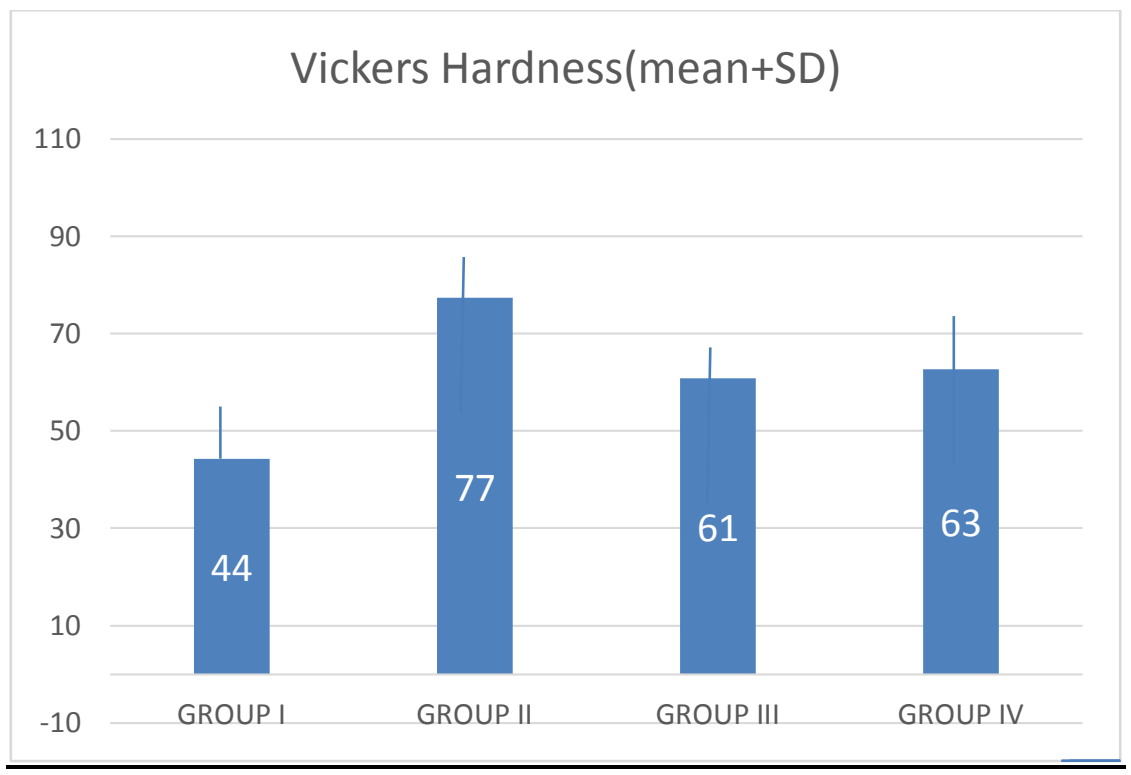

1. The mean hardness of Group 2(cention $\mathrm{N}$ ) was found to be highest among all the four groups and the mean hardness score of Group 1(Glass ionomer cement) is lowest among all the groups.

2. As per the hardness of Cention $\mathrm{N}$ (group 2) was significantly higher than rest of the groups. As per the single factor anova test the $\mathrm{p}$ value is less than $0.0001 \%$ (significance level). 
3. The hardness of type II glass ionomer cement (Group 1) was significantly lower than the rest.The p value is again less that $0.0001 \%$.

4. At an individual level, there was significant diffreence between Cention N(Group 2), the highest hardness group and silver amalgam (Group 4), the 2nd highest hardness group.The p value was less than $0.001 \%$.

5. At an individual level, there was significant diffreence between type II glass ionomer cement (Group 1), the lowest hardness group and nanohybrid composite resisn (Group 3),the 2nd lowest hardness group. The $\mathrm{p}$ value was less than $0.001 \%$.

\section{Discussion:-}

Cention $\mathrm{N}$ showed the highest hardness values among all the other materials. Probably their increased microhardness is related to the nanoparticle size of inorganic filling.

Hardness is defined as the resistance of a material to indentation or penetration. It has been used to predict the wear resistance of a material and its ability to abrade or be abraded by opposing tooth structures. [8] Hardness is often expressed as a percentage of the surface hardness which is considered $100 \%$. Experience has shown that the simple hardness measures (top and bottom) correspond well to the more thorough hardness profile measurements.[9] It is generally accepted that an adequate depth of cure has been achieved if the bottom hardness corresponds to at least $80 \%$ of the surface hardness. [10]

To define depth of cure based on top and bottom hardness measurements, it is common to calculate the ratio of bottom/top hardness, and give an arbitrary minimum value for this ratio. In order to consider the bottom surface as adequately cured, values of 0.80 and 0.85 have often been used. [5]

Dental materials to make minimal invasive treatments due to their aesthetics, easy handling, biocompatibility and adhesive properties; however, easy discoloration during their long term in the oral cavity and poor marginal sealing are the main disadvantages of their use and directly related to their composition and mechanical properties such as microhardness. [11] On the other hand, the oral environment is under constant $\mathrm{pH}$ and temperature cycles that can alter the organic and inorganic matrix of composite resins particles resulting in filtration and reducing their durability in the mouth. [7] While physical and mechanical properties of these materials may be significantly altered by the effects of solvent uptake and component elution, the greatest concerns are the short-term release of unreacted components and the long-term elution of degradation products in the oral cavity, both of which should be strongly considered during restorative material development.[12]

Glass ionomers are distinguished by their chemical bond to tooth structure, achieved via an exchange of ions arising from both the tooth and restoration. Etching of the enamel or dentin with phosphoric acid is not necessary. [13] Despite their reasonable clinical performance in terms of retention, glass ionomers are usually far less esthetic than composite. Glass ionomers combine the technologies and chemistry of silicate and zinc polycarboxylate materials so as to incorporate the desirable characteristics of both. [14] The fluoroaluminosilicate glass filler is ion-leachable but avoids the susceptibility to dissolution (a disadvantage in silicates) by substituting phosphoric acid with the polymeric carboxylic acids of zinc polycarboxylate materials. It has been found that silver amalgam and composite resin could withstand acidic solutions better than glass ionomer cement. [1]

Dental amalgam is a mixture of mercury, silver, tin and copper. Mercury, which makes up about 50 percent of the compound, is necessary to bind the metals together to provide a strong, hard, durable filling. [15] After years of research, mercury continues to be the only element that will bind these metals together in such a way that it can be easily manipulated to fill a cavity. In a study Amalgam, showed higher maximum stress and strain values than enamel. Hardness of high copper alloy amalgam showed greater values in many studies. But it has been found that the hardness of peripheral amalgam, especially at the marginal region, is obviously small compared to that at the central region. [16]

Resin composites are widely used in restorative dentistry and specifically in posterior restorations, putting the material under constant masticatory stresses. [17] Resin composites with better mechanical properties have been developed over these years. The hardness of composite resins is directly related to the conversion rate of polymerization depending on polymerization time, distance of polymerization light, irradiation power, and the type of material at the tip of the energy source. [18] However, a very powerful energy irradiation source can alter the 
polymerization contraction resulting in a poor marginal sealing and microfiltration. Stratification layers higher than $2 \mathrm{~mm}$ can partially polymerized, affecting the hardness of the material and increasing the risk for fracture. [19]

Due to the fact that Cention $\mathrm{N}$ is self-curing, the curing depth is theoretically unlimited. Cention $\mathrm{N}$ is a full volume replacement material, designed to be applied quickly and conveniently in bulk. In this context, it is important that the material exhibit low polymerization shrinkage and low shrinkage force.[1]

Cention $\mathrm{N}$ includes a special patented filler (partially functionalized by silanes) which keeps shrinkage stress to a minimum. This Isofiller, which is also used in Tetric N-Ceram Bulk Fill, acts as a shrinkage stress reliever which minimizes shrinkage force, whereas the organic/inorganic ratio as well as the monomer composition of the material, is responsible for the low volumetric shrinkage.

Fillers are responsible for imparting restorative materials with the adequate strength to withstand the stresses and strains of the oral cavity and to achieve acceptable clinical longevity. [20] The filler composition of Cention $\mathrm{N}$ is found in the Cention N Powder. The fillers of Cention $\mathrm{N}$ were chosen to achieve strength but also to obtain the desired handling characteristics of the mixed material. All the fillers therefore (except ytterbium trifluoride) are surface-modified to ensure wettability by the liquid and incorporation into the polymer matrix.

The Vickers hardness test utilises a diamond pyramid shaped indenter that is ground in the form of a squared pyramid with an angle of $136^{\circ}$ between faces and the depth of indentation is about $1 / 7$ of the resulting impression's diagonal length. Specimens are usually prepared in cylindrical moulds and the hardness at the top and bottom of the cylinder is measured to obtain a simple single hardness measured. [21]

Limitations of this study: In this study, only one physical property was tested on a limited number of materials polymerized with one type of unit. More research involving the use of other materials and multiple combinations of polymerization modes is warranted. The results apply only to these materials and shades under the testing conditions stated. The test were performed in cylindrical moulds, different results may be obtain in case of tooth preparation. Another limitation is that different types of cavity designs are required for different restorative materials, which is an important factor in evaluation hardness of a restoration. More research involving the use of the materials and multiple combinations of polymerization modes and various techniques are needed.

\section{Conclusion:-}

Within the limitations of this study, the following conclusions were drawn:-

According to our results, the microhardness of restorative materials could withstand the masticatory forces in the clinical context.

However, Cention $\mathrm{N}$ showed better microhardness properties becoming a more clinically suitable option for minimal invasive treatments.

\section{References:-}

1. Scientific Documentation: Cention N Ivoclar Vivadent AG Research \& Development Scientific Service october 2016

2. Mohamed El-Nawawy et al(2012), Depth of Cure and Microhardness of Nanofilled, Packable and Hybrid Dental Composite Resins. American Journal of Biomedical Engineering 2012, 2(6): 241-250

3. Martin S. Spiller (2012), Dental Composites: A Comprehensive Review, Academy of dental learning and osha training.

4. Ugur Erdemir et al (2013), Surface hardness evaluation of different composite resin materials: influence of sports and energy drinks immersion after a short-term period, Journal of Applied Oral Sci. 2013;21(2):124-31

5. Poggio C et al (2012), Evaluation of Vickers hardness and depth of cure of six composite resins photoactivated with different polymerization modes, Journal of Conservative Dentistry, Jul-Sep 2012, Vol 15, Issue

6. Nidal w. Elshereksi et al(2017), effect of nanobarium titanate addition on the surface characteristics of denture base resin, international journal of mechanical and production engineering (ijmpe) , pp. 1-8, volume-5,issue-5

7. René García-Contreras et al (2015), Vickers microhardness comparison of 4 composite resins with different types of filler. Journal of Oral Research 2015; 4(5): 313-320. 
8. Abdul Mujeeb et al (2014) In vitro Evaluation of Topical Fluoride ph and their Effect on Surface Hardness of Composite Resin-based Restorative Materials, The Journal of Contemporary Dental Practice, March-April 2014;15(2):190-194

9. Khulood Al-Mansour et al (2015) Curing depth of bulk- fill composites- an in- vitro study Pakistan Oral \& Dental Journal Vol 35, No. 2 (June 2015)

10. Scientific Documentation Tetric Evo Ceram Bulk Fill Ivoclar Vivadent AG Research \& Development Scientific Service March 2014

11. Mohammed Almuhaiza (2016),Glass-ionomer cements in restorative dentistry: A critical appraisal, The journal of contemporary dental practice, April 2016;17(4):331-336

12. Jack L. Ferracane (2006), Hygroscopic and hydrolytic effects in dental polymer networks, Dental Materials (2006) 22, 211-222

13. Maryam Khoroushi et al(2013) A review of glass-ionomers: From conventional glass-ionomer to bioactive glass-ionomer, Dental Research Journal (Isfahan). 2013 Jul-Aug; 10(4): 411-420.

14. John Paul (2015), Dental Cements - A Review to Proper Selection, Volume 4 Number 2 (2015) pp. 659-669

15. Delta Dental: Dental Amalgam facts

16. Arvind Shenoy (2008), Is it the end of the road for dental amalgam? A critical review, Journal of Conservative Dentistry, Jul-Sept 2008, Vol 1, Issue 3

17. Mohamed El-Nawawy et al (2012), Depth of Cure and Microhardness of Nanofilled, Packable and Hybrid Dental Composite Resins, American Journal of Biomedical Engineering 2012, 2(6): 241-250

18. Camila Sabatini (2013) et al, Comparative study of surface microhardness of methacrylate-based composite resins polymerized with light-emitting diodes and halogen, European Journal of Dentistry, Vol 7,Issue 3, JulSep 2013

19. Katia M. Rode (2009) et al, Micro-hardness evaluation of a micro-hybrid composite resin light cured with halogen light, light-emitting diode and argon ion laser, Lasers Med Sci (2009) 24:87-92

20. Parag K. Shah et al(2014), Role of filler and functional group conversion in the evolution of properties in polymeric dental restoratives, Dental Material. 2014 May ; 30(5): 586-593.

21. David A. Lazarchik (2007) Hardness comparison of bulk-filled/ transtooth and incremental-filled/ occlusally irradiated composite resins, The Journal of Prosthetic Dentistry,2007; Volume 98 Issue 2 